\title{
NONMETRIZABLE HEREDITARILY LINDELÖF SPACES WITH POINT-COUNTABLE BASES FROM CH
}

\author{
ERIC K. VAN DOUWEN, FRANKLIN D. TALL ${ }^{1}$ AND WILLIAM A. R. WEISS ${ }^{2}$
}

\begin{abstract}
A nonmetrizable hereditarily Lindelöf space with a point-countable base is obtained by using the continuum hypothesis to construct a Lusin subspace of a countable chain condition first countable nonseparable Baire space.
\end{abstract}

There has lately been much interest in the relationship between hereditary Lindelöfness and hereditary separability within the class of regular spaces. See e.g. $\left[\mathbf{R u}_{\mathbf{2}}\right]$. We shall assume all spaces regular. The question is whether either property entails the other, possibly with other conditions on the spaces, possibly with the assumption of various consistent set-theoretic axioms. A rule of thumb which has emerged from recent investigations is the kinds of counterexamples constructed from a Souslin line can also be obtained from the assumption of the continuum hypothesis (henceforth called $\mathrm{CH}$ ). For example, a Souslin line is hereditarily Lindelöf nonseparable, while such spaces were later obtained using $\mathrm{CH}[\mathbf{H J}]$. Similarly, hereditarily separable non-Lindelöf spaces were first constructed from a Souslin line $\left[\mathbf{R} \mathbf{u}_{\mathbf{1}}\right]$, then from $\mathrm{CH}[\mathbf{H J}]$. We provide another confirmation of this rule by using $\mathrm{CH}$ to construct a hereditarily Lindelöf space with a point-countable base which is not metrizable (and hence not separable). Such spaces were obtained from Souslin lines in $[\mathbf{P}]$ and $[\mathbf{B}]$. See $\left[\mathbf{T}_{\mathbf{1}}\right]$ for further background.

Our construction may be outlined as follows. A Lusin space is an uncountable space such that every nowhere dense set is countable. We observe that dense Lusin subspaces of (first countable) countable chain condition nonseparable spaces are (first countable) hereditarily Lindelöf nonseparable. As in [ $\mathbf{T}_{\mathbf{1}}$ ], a first countable example may be converted into one with a point-countable base. By examining the classical proof (see e.g. [Ku]) that $\mathrm{CH}$ entails the existence of Lusin subspaces of the real line, we see that such subspaces exist in countable chain condition (henceforth CCC) first countable spaces without isolated points satisfying the Baire category theorem. The proof is completed

Received by the editors November 28, 1975.

AMS (MOS) subject classifications (1970). Primary 54A25, 54D20, 54E35; Secondary 02K05, 02K25, 04A 30.

Key words and phrases. Hereditarily Lindelö nonseparable, point-countable base, continuum hypothesis, Lusin space, Pixley-Roy topology.

1 The second author acknowledges support from Grant A-7354 of the National Research Council of Canada.

2 The third author acknowledges support from NRC postgraduate and postdoctoral fellowships. 
by exhibiting a nonseparable example of the latter type of space.

First we consider Lusin spaces.

Definition. A space is Baire if no nonempty open set is the union of countably many nowhere dense sets.

Definition. $\pi(X)$ is the least cardinal of a collection (called a $\pi$-base) of nonempty open sets such that every nonempty open set includes one. $c(X)$ is the sup of cardinals of collections of disjoint open subsets of $X$.

Theorem 1. Assume CH. If $X$ is uncountable CCC Baire without isolated points and $\pi(X) \leqslant 2^{\aleph_{0}}, X$ includes a dense Lusin subspace.

COROllary. Assume CH. Every uncountable CCC first countable Baire space without isolated points includes a dense Lusin subspace.

Proof. The Corollary follows since CCC first countable spaces have cardinality and hence weight $\leqslant 2^{\aleph_{0}}[\mathbf{J}]$. To prove the theorem, first observe that every nowhere dense subset of $X$ is included in the complement of the union of a maximal disjoint collection of elements of the $\pi$-base. Each such complement is nowhere dense and there are $\leqslant\left(2^{\kappa_{0}}\right)^{\kappa_{0}}=2^{\kappa_{0}}$ of them, say $\left\{F_{\alpha}\right\}_{\alpha<\omega_{1}}$. If we can construct a dense uncountable $Y \subseteq X$ with countable intersection with each $F_{\alpha}$, we are done. Let $\left\{P_{\alpha}\right\}_{\alpha<\omega_{1}}$ be a $\pi$-base for $X$. Suppose for each $\beta<\alpha<\omega_{1}$, a $y_{\beta}$ has been chosen in $P_{\beta}-\cup_{\gamma<\beta} F_{\gamma}$. Pick

$$
y_{\alpha} \in P_{\alpha}-\left(\bigcup_{\beta<\alpha} F_{\beta} \cup\left\{y_{\beta}: \beta<\alpha\right\}\right) \text {. }
$$

Then $\left\{y_{\alpha}\right\}_{\alpha<\omega_{1}}$ is dense Lusin.

Note that this argument does not require the $P_{\alpha}$ 's to be distinct and thus encompasses the case when $X$ is the real line. If $X$ is CCC nonseparable, the isolated points clause can be dropped if the Lusin set is not required to be dense. As the theorem stands, the isolated points clause is necessary:

EXAMPLE. A locally compact first countable Hausdorff space with a countable dense set of isolated points and no Lusin subspace.

Let $X$ consist of the real line plus those points in the upper half plane with both coordinates rational. The rational points are isolated. For each point on the line choose a fixed sequence of rational points converging to it and let neighbourhoods of the point be tails of the sequence.

(We have recently learned that Theorem 1 and other interesting results concerning Lusin spaces were announced in [A $\check{\mathbf{S}}$ ]. White [Wh] was the first to note Lusin subspaces yielded hereditarily Lindelöf spaces.)

It is perhaps of interest that a variation of the conclusion of Theorem 1 is actually equivalent to $\mathrm{CH}$.

THEOREM 2. CH iff every uncountable CCC Baire space of $\pi$-weight $\leqslant 2^{\kappa_{0}}$ without isolated points has a Lusin subspace of power $2^{\kappa_{0}}$.

Proof. Assuming the right-hand side, both the real line with the usual topology and the real line with the density topology $\left[\mathbf{T}_{\mathbf{3}}\right]$ have Lusin subspaces 
of power continuum. In the latter topology, a Lusin subspace is a Sierpinski set, i.e. a set of reals such that every subset of measure zero is countable. By $[\mathbf{R}]$, the simultaneous existence of a Lusin subspace of the reals and a Sierpiński set of reals, at least one of power continuum, implies $\mathrm{CH}$.

Now to construct the required Baire space.

THEOREM 3. There exists a first countable zero-dimensional CCC Baire space in which no nonempty open subset is separable.

Construction. The example is the space $t h$ of all nonempty compact nowhere dense subsets of $\mathbf{R}$ (the real line), equipped with the Pixley-Roy topology (defined below). This topology for a space of subsets is studied in [vD $\mathbf{v D}_{1}$ and is motivated by an example of Pixley and Roy [PR]. Because points, or subsets, of $\mathcal{K}$ are subsets, or families of subsets of $\mathbf{R}$, they are denoted by Roman capitals, or script capitals, respectively; families of subsets of $\mathcal{K}$ are denoted by German capitals.

For any subsets $F$ and $G$ of $\mathbf{R}$ we define a subset $[F, G]$ of $\mathscr{K}$ by

$$
[F, G]=\{K \in \mathscr{K}: F \subseteq K \subseteq G\} .
$$

The Pixley-Roy topology on $\mathscr{K}$ is defined by taking for each $K \in \mathfrak{K}$, all sets of the form $[K, U]$, where $U$ is a neighbourhood of $K$ in $\mathbf{R}$, to be a neighbourhood base for $K$ in $\mathscr{K}$. Since each $K \in \mathcal{K}$ is a compact subset of $\mathbf{R}$, it is easily verified that $\mathscr{K}$ is first countable. It is also easy to verify that each set of the form $[F, G]$ is closed in $\mathscr{K}$. Since $\{K\}=[K, K]$ for $K \in \mathfrak{h}$, it follows that $\mathscr{K}$ is a zero-dimensional $T_{1}$-space.

Recall that a family of sets is centered if each finite subfamily has nonempty intersection. We call a family $\sigma$-centered if it is the union of countably many centered subfamilies. In order to show that $\mathscr{K}$ is a CCC space, we prove the stronger result that $\mathcal{K}$ has a $\sigma$-centered base. In $\left[\mathbf{v D}_{2}\right]$ it is shown that this is equivalent to $b \mathcal{K}$ being separable for some (equivalently: for every) Hausdorff compactification $b \mathcal{K}$ of $\mathcal{K}$. Let $\mathcal{O}$ f be any countable base for $\mathbf{R}$ such that $V \cup W \in \mathscr{W}$ for any $V, W \in \mathscr{W}$. Then for each compact $K \subseteq \mathbf{R}$ and each neighbourhood $U$ of $K$ in $\mathbf{R}$ there is a $W \in \mathscr{Q} \int$ such that $K \subseteq W \subseteq U$. So, if we define for each $W \in \mathscr{q} \mid$ a collection $₫(W)$ by

$$
\mathfrak{S}(W)=\{[K, W]: K \in \mathscr{K} \text { and } K \subseteq W\},
$$

then the family $\mathbb{E}=\cup\{\mathbb{E}(W): W \in \mathscr{W}\}$ is a base for $\mathscr{K}$. But $\mathbb{E}$ is $\sigma$-centered since $U \cap V \in \mathbb{S}(W)$ whenever $U, V \in \mathbb{S}(W)$, and $\varnothing \notin \mathbb{S}(W)$, for each $W \in \mathcal{Q}$.

In order to prove that $\mathcal{K}$ is a Baire space, it suffices to show that $\mathscr{K}$ satisfies the following "completeness condition", which resembles Čech completeness:

There is a sequence $\left\{\mathscr{B}_{n}: n<\omega\right\}$ of families of (closed) subsets of $\mathfrak{K}$ satisfying:

(1) for each $n<\omega$, for each $K \in \mathcal{K}$ and for each neighbourhood $\mathscr{Q}$ of $K$ in $\mathscr{K}$, there is a $\mathscr{B} \in \mathfrak{B}_{n}$ such that $K \subseteq$ int $_{\mathscr{K}} \mathscr{G}$ and $\mathscr{B} \subseteq \mathscr{Q}$; and 
(2) if $\mathfrak{A} \subseteq \cup\left\{\mathfrak{B}_{n}: n<\omega\right\}$ is any centered family such that $\mathfrak{A} \cap \mathfrak{B}_{n} \neq \varnothing$ for each $n<\omega$, then $\cap \mathfrak{A} \neq \varnothing$.

If $\left\{B_{n}: n<\omega\right\}$ is any base for $\mathbf{R}$, then we define the families $\mathfrak{B}_{n}$ by

$$
\mathfrak{B}_{n}=\left\{[K, F]: K \in \mathscr{K}, F \subseteq \mathbf{R} \text { is compact, } K \subseteq \operatorname{Int}_{\mathbf{R}} F, B_{n}-F \neq \varnothing\right\} .
$$

Then (1) is an immediate consequence of the fact that each member of $\mathcal{K}$ is a compact nowhere dense subset of $\mathbf{R}$. Next let $\mathfrak{A}=\left\{\left[K_{i}, F_{i}\right]: i \in I\right\}$ be any centered subfamily of $\cup\left\{\mathfrak{B}_{n}: n<\omega\right\}$ such that $\mathfrak{A} \cap \mathfrak{B}_{n} \neq \varnothing$ for each $n<\omega$. Define $F=\cap\left\{F_{i}: i \in I\right\}$. Then for each $n<\omega$ we have that $B_{n}-F \neq \varnothing$ since $\mathfrak{U} \cap \mathfrak{B}_{n} \neq \varnothing$. Therefore $F$ is a nowhere dense subset of $\mathbf{R}$ which clearly is compact. Next we observe that for any $i, j \in I$ we have that $K_{i} \subseteq F_{j}$, since

$$
\varnothing \neq\left[K_{i}, F_{i}\right] \cap\left[K_{j}, F_{j}\right]=\left[K_{i} \cup K_{j}, F_{i} \cap F_{j}\right] .
$$

Therefore $K_{i} \subseteq F$ for all $i \in I$. But this shows that $F \neq \varnothing$, hence $F \in \mathfrak{K}$, and also that $K_{i} \subseteq F \subseteq F_{i}$ for all $i \in I$, so $F \in \cap \mathfrak{A}$.

We now show that no nonempty open subset of $\mathfrak{h}$ is separable. Let $K$ be any member of $\mathscr{K}$, let $U$ be any open subset of $\mathbf{R}$ which contains $K$, and let $\mathcal{D}$ be a dense subset of $[K, U]$. For each $x \in U$ the set $K \cup\{x\}$ belongs to $\mathcal{K}$, hence $K \cup\{x\} \subseteq D \subseteq U$ for some $D \in \mathscr{D}$. Therefore $U=\cup \mathscr{D}$. Since each member of $\mathscr{D}$ is nowhere dense, it follows that $\mathscr{D}$ is not countable.

It is of interest to note that $\mathscr{K}$ is submetrizable (i.e. $\mathscr{K}$ has a weaker metrizable topology). Indeed, let $\subseteq$ be the family consisting of all sets of the form $\{K \in \mathscr{K}: K \cap U \neq \varnothing\}$ or $\{K \in \mathscr{K}: K \subseteq U\}$, where $U$ is open in $\mathbf{R}$. Each member of $\subseteq$ is open in $\mathscr{K}$, but $\subseteq$ is a subbase for the Vietoris topology on $\mathscr{K}$. This topology is second countable and hence metrizable [M]. It follows that $\mathcal{K}$ has a $G_{\delta}$-diagonal and a countable separating open cover. Hodel $[\mathbf{H}]$ has shown that a regular quasi-complete CCC Baire space $X$ is separable provided it has a $G_{\delta}$-diagonal or a point-countable separating open cover and notes that the requirement that $X$ be Baire is necessary. $\mathscr{K}$ establishes that quasi-completeness is also essential.

$\mathcal{K}$ is in fact cometrizable [AP]. This observation is used in $\left[\mathbf{T}_{\mathbf{4}}\right]$ to construct, assuming Martin's Axiom plus not $\mathrm{CH}$, a dense Baire normal noncollectionwise Hausdorff subspace of $\mathcal{K}$ having caliber $\aleph_{1}$.

We now have a CCC nonseparable first countable 0-dimensional Baire space $\mathcal{K}$ without isolated points. Let $\mathcal{L}$ be a dense Lusin subspace of $\mathcal{K}$. Then $\mathcal{L}$ is CCC nonseparable. It is easily verified that CCC spaces in which nowhere dense sets are Lindelöf are hereditarily Lindelöf. As in $\left[\mathbf{T}_{\mathbf{1}}\right]$, take $Z=\left\{z_{\alpha}\right\}_{\alpha<\omega_{1}}, z_{\alpha} \notin \overline{\left\{z_{\beta}: \beta<\alpha\right\}}$. Take a neighbourhood base $\left\{U_{\alpha, i}\right\}_{i<\omega}$ for $z_{\alpha}$ such that for each $\alpha$ and each $i, U_{\alpha, i} \cap\left\{z_{\beta}: \beta<\alpha\right\}=\varnothing$. Then $\left\{U_{\alpha, i}\right.$ : $\left.\alpha<\omega_{1}, i<\omega\right\}$ is a point-countable base for $Z$, which thus is the desired nonmetrizable 0 -dimensional hereditarily Lindelöf space with a point-countable base.

$Z$ also has a $G_{\delta}$-diagonal. The example in $\left[\mathbf{T}_{1}\right]$ does not have a $G_{\delta}$-diagonal 
because it is linearly orderable, and a linearly orderable space with a $G_{\delta}$-diagonal is metrizable [ $\left.\mathbf{L}\right]$.

It should be remarked that it was the requirement that our space be first countable that occasioned some effort to find an example. We can give a quick proof from $\mathrm{CH}$ of the existence of a hereditarily Lindelöf 0 -dimensional nonseparable space by constructing a Lusin subspace of $\left\{f \in 2^{\omega_{1}}: f(\alpha)=0\right.$ except for countably many $\alpha$ \}. The latter space (with the subspace topology inherited from the product) is easily seen to be CCC, nonseparable, 0-dimensional, Baire, and to have $\pi$-weight $\kappa_{1}$. See e.g. $\left[\mathbf{T}_{2}\right]$. Other spaces that are CCC, nonseparable, Baire, and have $\pi$-weight $\leqslant 2^{\kappa_{0}}$ are, for example, the density topology and the Stone space of the reduced measure algebra.

We take this opportunity to mention a couple of results that, if observed earlier, would have appeared in $\left[\mathbf{T}_{\mathbf{1}}\right]$.

THEOREM 3. If there is a countably compact perfectly normal space which is not hereditarily separable, then there is a nonmetrizable hereditarily Lindelöf space with a point-countable base.

Proof. Countably compact perfectly normal spaces are first countable, and by [St] have no uncountable discrete subspace. Apply Theorem 2 of [ $\mathbf{T}_{\mathbf{1}}$ ].

THEOREM 4. If there is a nonmetrizable hereditarily Lindelöf space with a point-countable base, there is a 0-dimensional one. If there is a nonseparable hereditarily Lindelöf space, there is a 0-dimensional one.

Proof. If $\mathrm{CH}$, our example will do. If not $\mathrm{CH}$, any completely regular space of cardinality $\kappa_{1}$ is 0 -dimensional. It is easy to see that if either of the spaces mentioned in the hypotheses of the theorem exists, then it has a subspace of power $\kappa_{1}$ with the same properties.

Jensen [DJ] has constructed a model of set theory in which there are no Souslin lines, but $\mathrm{CH}$ holds. In this model there is a nonmetrizable hereditarily Lindelöf space with a point-countable base, but there is none which is linearly ordered.

Under the assumption of Martin's Axiom plus not $\mathrm{CH}$, every countably compact perfectly normal space is hereditarily separable [W]. It remains open whether this assumption ensures the metrizability of hereditarily Lindelöf spaces with point-countable bases. However, after completing this paper, we learned that Kunen [K] had recently proved

THEOREM 5. If Martin's Axiom plus not $C H$ is assumed, there are no CCC Lusin spaces.

In the light of another observation of Kunen-namely that Souslin lines have dense Lusin subspaces-the construction of a nonmetrizable hereditarily Lindelöf space with a point-countable base from a Souslin line can be regarded as a special case of the method developed in this paper.

Subsequent to our work, Kunen constructed from $\mathrm{CH}$ a compact perfectly 
normal nonseparable space. By Theorem 3 this yields another nonmetrizable hereditarily Lindelöf space with a point-countable base.

Using the new axiom $\mathrm{BACH}$, the second author $\left[\mathrm{T}_{\mathbf{5}}\right]$ has constructed a dense Lusin subspace of $2^{\kappa}$, for regular $\kappa<2^{\kappa_{1}}$.

Subspaces of the resulting hereditarily Lindelöf nonseparable space all have weight either $\kappa$ or $\leqslant \kappa_{1}$.

\section{REFERENCES}

[AP] K. Alster and T. Przymusiński, Normality and Martin's Axiom, Fund. Math. 91 (1976), 123-131.

[AS̆] G. P. Amirdžanov and B. Ė. Šapirovskii, Everywhere-dense subsets of topological spaces, Dokl. Akad. Nauk SSSR 214 (1974), 249-252 = Soviet Math. Dokl. 15 (1974), 87-92. MR 49 \#7972.

[B] H. R. Bennett, Quasi-developable spaces, Dissertation, Arizona State Univ., Tempe, Arizona, 1968.

[DJ] K. Devlin and H. Johnsbraten, The Souslin problem, Lecture Notes in Math., vol. 405, Springer-Verlag, New York, 1975.

[VD, E. K. Van Douwen, The Pixley-Roy topology on spaces of subsets (preprint).

$\left[\mathrm{vD}_{2}\right] \ldots$, Density of compactifications (preprint).

[H] R. E. Hodel, Metrizability of topological spaces, Pacific J. Math. 55 (1974), 441-459. MR 51 \#6747.

[HJ] A. Hajnal and I. Juhász, On hereditarily $\alpha$-Lindelöf and $\alpha$-separable spaces. II, Fund. Math. 81 (1973/74), 147-158. MR 49 \#1478.

[J] I. Juhász, Cardinal functions in topology, Mathematical Centre Tracts, No. 34, Mathematisch Centrum, Amsterdam, 1971. MR 49 \#4778.

[K] K. Kunen, Luzin spaces (preprint).

[Ku] K. Kuratowski, Topology, Vol. I, Academic Press, New York, 1966. MR 36 \#840.

[L] D. J. Lutzer, A metrization theorem for linearly orderable spaces, Proc. Amer. Math. Soc. 22 (1969), 557-558. MR 40 \#2012.

[M] E. A. Michael, Topologies on spaces of subsets, Trans. Amer. Math. Soc. 71 (1951), 152-182. MR 13, 54.

[P] V. I. Ponomarev, The metrizability of a finally compact p-space with a point countable base, Dokl. Akad. Nauk SSSR 174 (1967), 1274-1277 = Soviet Math. Dokl. 8 (1967), 765-768. MR 35 \#7298.

[PR] C. Pixley and P. Roy, Uncompletable Moore spaces, Proc. Auburn Topology Conf. (Auburn, Alabama, 1969), pp. 75-85.

[R] F. Rothberger, Eine äquivalenz zwischen der Kontinuumhypothese und der Existenz der Lusinschen und Sierpinskischen Mengen, Fund. Math 30 (1938), 215-217.

$\left[\mathbf{R u} \mathbf{u}_{1}\right] \mathrm{M}$. E. Rudin, $\boldsymbol{A}$ normal hereditarily separable non-Lindelöf space, Illinois J. Math. 16 (1972), 621-626. MR 46 \#8173.

$\left[\mathbf{R u}_{2}\right] \ldots$, Lectures on set-theoretic topology, CBMS Regional Conf. Ser. in Math., no. 23, Amer. Math. Soc., Providence, R.I., 1975. MR 51 \# 4128.

[St] R. M. Stephenson, Jr., Discrete subsets of perfectly normal spaces, Proc. Amer. Math. Soc. 34 (1972), 605-608. MR 45 \#5944.

[ $\left.\mathbf{T}_{1}\right]$ F. D. Tall, On the existence of non-metrizable hereditarily Lindelö spaces with point-countable bases, Duke Math. J. 41 (1974), 299-304.

$\left[\mathbf{T}_{2}\right] \ldots$, The countable chain condition versus separability-applications of Martin's Axiom, General Topology and Appl. 4 (1974), 315-340.

$\left[T_{3}\right] \ldots$, The density topology, Pacific J. Math. 62 (1976), 275-284.

$\left[\mathrm{T}_{4}\right]$, First countable spaces with caliber $\mathrm{N}_{1}$ may or may not be separable, Set-Theoretic Topology (G. M. Reed, Editor), Academic Press, New York, 1977.

$\left[\mathbf{T}_{\mathbf{5}}\right] \ldots$, Some topological applications of a generalized Martin's Axiom (in preparation). 
[W] W. A. R. Weiss, Some applications of set theory to topology, Thesis, Univ. of Toronto, Toronto, Canada, 1975.

[Wh] H. E. White, Jr., Topological spaces in which Blumberg's theorem holds, Proc. Amer. Math. Soc. 44 (1974), 454-462. MR 49 \#6130.

Institute For Medicine and Mathematics, Morton Hall, Ohio University, Athens, Оніо 45701

Department of Mathematics, University of Toronto, Toronto, Ontario, Canada M5S $1 \mathrm{~A} 1$

Department of Mathematics, Erindale College, University of Toronto, Mississauga, Ontario, Canada L5L 1 C6 